\section{HIGH-DIMENSIONAL IMAGE CYTOMETRY REVEALS SPATIALLY ORGANIZED TUMOR-IMMUNE MICROENVIRONMENT IN HEPATOCELLULAR CARCINOMA}

Haoyang Mi*, Won Jin Ho, Mark Yarchoan, Aleksander S Popel. Johns Hopkins University Baltimore, MD, United States

Background Structured and spatial-nuanced interactions between components in tumor microenvironment (TME) regulates the efficacy of anti-tumor regimens. Insights into this orchestrated behavior in therapeutic responders and non-responders will facilitate immunotherapies. High-multiplex imaging and spatial statistics enable deep profiling of TMEs by simultaneous arraying cell phenotypes and locations. In this study, we quantified the landscape of TMEs from neoadjuvant cabozantinib and nivolumab administered locally advanced hepatocellular carcinoma (HCC) biospecimen.

Methods 14 patients with HCC were treated with the combination of cabozantinib and nivolumab through the Johns Hopkins Sidney Kimmel Comprehensive Cancer Center. Among them, 12 patients (5 responders +7 non-responders) underwent successful margin negative resection and are subjects to tissue microarray (TMAs) construction containing 37 representative tumor region cores. Using the TMAs, we performed imaging mass cytometry (IMC) with a panel of 27-cell lineage and functional markers (figure 1). All multiplexed images were then segmented to generate a single-cell dataset that enables (1) tumor-immune compartment analysis and (2) cell community analysis based on graph-embedding technology. Results from these hierarchies are merged to response-associated biological process patterns.

Results Image processing on 37 multiplexed images discriminated 59,453 cells and then clustered into 17 cell types. Multi-level spatial quantification revealed distinct TME arrangements across cores from responders (R) and non-responders (NR): compartment analysis showed that at immunetumor boundaries from NR, PD-L1 level on tumor cells is significantly higher than remote regions; however, Granzyme B level is lower (figure 2B). We also identified the proximity of $\mathrm{CD} 8+\mathrm{T}$ cells to a subset of macrophages - Arginase $1 \mathrm{hi}$ CD163- macrophages (hazard macrophage) and CD4+ T cells, is a prognostic biomarker to neoadjuvant therapy (figure $3 \mathrm{~A}$ and $3 \mathrm{~B})$. In-depth cell community analysis extracted cell-cell interaction networks based on spatial proximity. Next, hierarchical clustering grouped all networks with similar components (cell types) into 8 community categories (CC). Using graph-embedding and correlation test, we observed that in NR, macrophage-enriched CC (MCC) and lymphocytesenriched CC (LCC) are strongly communicating with tumor $\mathrm{CC}$; whereas in $\mathrm{R}$, such communications were weakened by the engagement between MCC and LCC (figure 3C).

Conclusions In conclusion, we employed an unbiased, quantitative spatial analysis to determine how tumor and immune components interact in responding and nonresponding HCC tumors. Based on our results, four immune-regulating factors are derived and summarized as a communication landscape (figure 4). The proposed framework represents a novel application of multiplexed imaging in translational medicine and has potential in initialization and validation of computational immuno-oncology models.

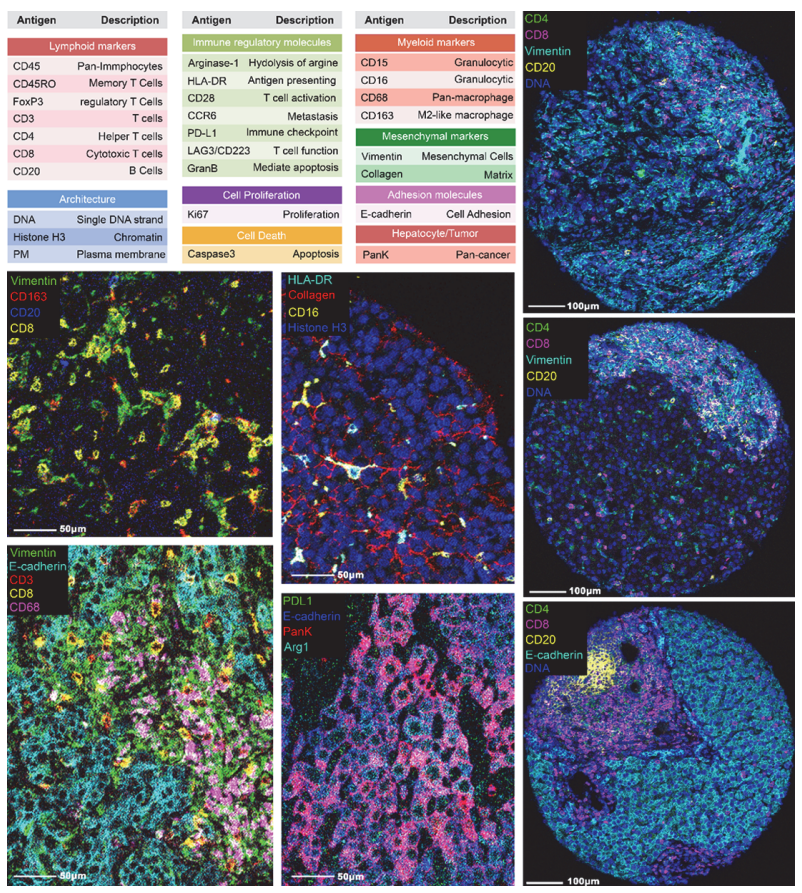

Abstract 825 Figure 1 A panel of 27 markers was used to stain the hepatocellular carcinoma tumor region cores and processed using IMC. The marker names and descriptions are included

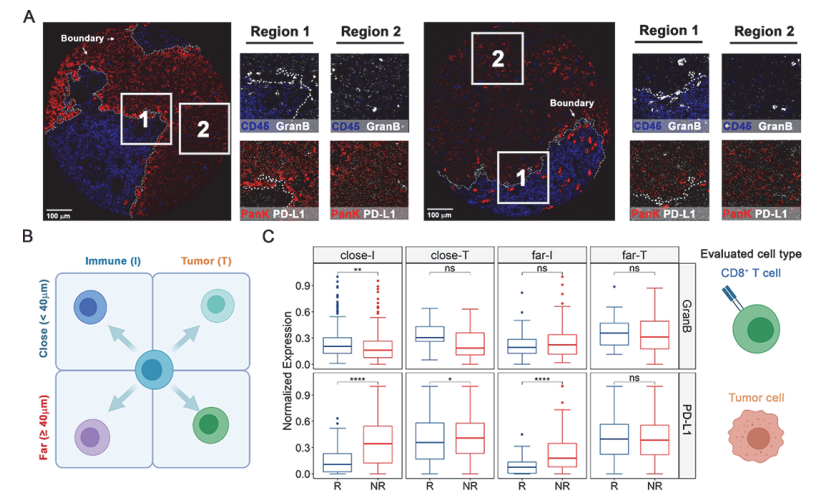

Abstract 825 Figure 2 (A) Color overlays of lineage proteins covering Pan-Keratin and CD45 (rainbow) and functional markers covering PD-L1 and Granzyme B (white) in whole tissue core and subregions. (B) and (C) Protein expression analytical strategy. For compartmentalized cores, functional marker expressions on target cells were examined adjacent and remote to tumor-immune border and truncated to treatment response criteria for comparisons 


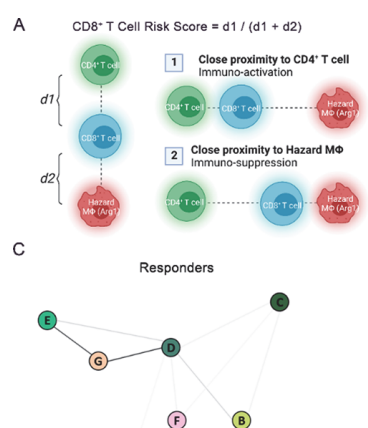

(A)

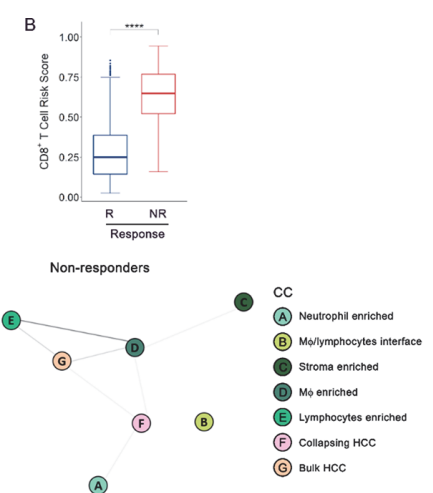

(A)

Abstract 825 Figure 3 (A) Diagram of CD8+ T cell RiskScore. Denote each CD8+ T cell to its nearest hazard macrophages as d1 and to its nearest CD4+ $T$ cell as $\mathrm{d} 2$, thus the RiskScore is formally computed by taking the proportion of $d 2$ to the combined distance of $d 1$ and $d 2$. (B) RiskScore on per-cell basis for responders and non-responders. (C) Cell community communication maps in tumor microenvironment associated with responders and non-responders
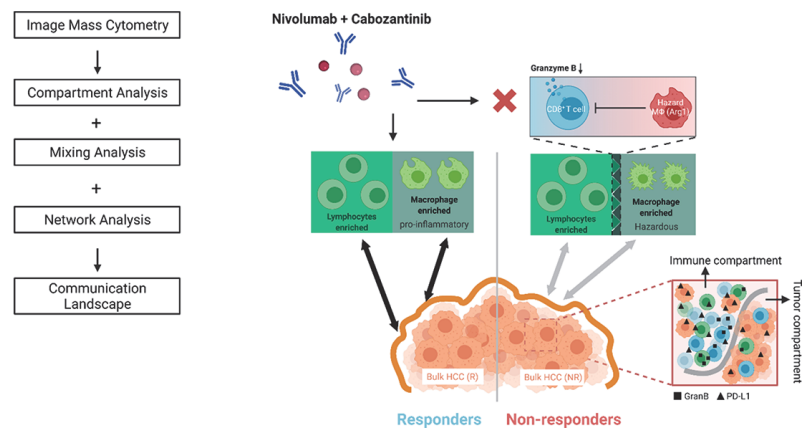

Abstract 825 Figure 4 The synergistic anti-tumor immunity of macrophages and lymphocytes favors cabozantinib and nivolumab; immune function regulators (i.e., GranB and PD-L1) were upregulated throughout the immune compartment in non-responders; close proximity to hazard macrophages and distance away from CD4+ T cells associate with poorer effector function of CD8+ T cells

Acknowledgements The authors acknowledge financial support from Bristol-Myers Squibb, Exelixis, the National Cancer Institute Specialized Program of Research Excellence (SPORE) in Gastrointestinal Cancers (P50 CA062924), the Passano Foundation, the National Institutes of Health (Grant No. U01CA212007 and R01CA138264) and Emerson Collective Cancer Research Fund (640183).

Ethics Approval The studies involving human participants were reviewed and approved by Institutional Review Board of the Johns Hopkins Medical Institutions.

Consent Written informed consent for participation was not required for this study in accordance with the national legislation and the institutional requirements.

http://dx.doi.org/10.1136/jitc-2021-SITC2021.825 